\title{
Ability to Propose Optimal Prosthetic Rehabilitation can be Improved by Discussion between the Dentist and Radiation Oncologist Regarding Upstream Dosimetry
}

\author{
Mélanie Rouers ${ }^{1}$ Fabien Bornert ${ }^{1}$ Pierre Truntzer ${ }^{2}$ \\ Delphine Antoni' ${ }^{2,3}$ Georges Noël' ${ }^{2,3}$ \\ ${ }^{1}$ Faculty of Dental Medicine, 1 Place de l'Hôpital, Strasbourg, France \\ 2University Radiation Department, Centre Paul Strauss, Strasbourg, \\ France \\ ${ }^{3}$ Strasbourg University, Radiobiology Lab, Centre Paul Strauss, \\ Strasbourg, France
}

Sarah Dubourg ${ }^{1}$ Cyrielle Bourrier ${ }^{2}$

Eur J Dent 2019;13:88-94

\begin{abstract}
Address for correspondence Georges Noël, MD, PhD. Strasbourg University, Radiobiology Lab, CNRS, IPHC UMR 7178, Centre Paul Strauss, UNICANCER, F-67000, Strasbourg, France (e-mail: gnoel@strasbourg.unicancer.fr).
\end{abstract}

\begin{abstract}
Objective Improvement of dental rehabilitation for patients who have undergone radiation therapy requires knowledge of the dose in the maxillary and mandible bones.

Materials and Methods Forty-three patients with head and neck cancers underwent evaluation for dental rehabilitation before radiation treatment dosimetry. The delivered dose to the maxilla and mandible was determined. From the dose data in the literature, three levels of risk of implant failure were defined. According to the delivered doses, the authors calculated the percentage of patients who could be fully rehabilitated with an implant, as proposed by the dentist before radiation planning.

Results Before dosimetry calculation, all of the completely edentulous arches and 94 partially edentulous (PESs) sextants could be optimally rehabilitated. After dose calculation, among the 14 arches of 7 patients who were completely edentulous, according to the mean and maximal delivered doses, 11 arches (78.6\%) and 7 arches (50\%) could receive an optimal prosthesis, respectively. For the three patients, who were PESs but with one arch that was completely edentulous, according to the mean and maximal delivered doses, one arch for each dose condition could receive an optimal prosthesis. Among the 94 PESs sextants, according to the mean and

Keywords

- dental care

- dental implant

- dental prosthesis

- head and neck cancer

- treatment planning maximal delivered doses, 41 (43.6\%) and 24 (25.5\%) sextants could receive an optimal prosthesis, respectively.

Conclusion By determining the sites of implantation before dosimetry, the radiation oncologist could shield specified areas, potentially improving the possibilities for dental rehabilitation. The dialogue between the dentist and the radiation oncologist can improve the possibilities for implants and decrease the risk of unsafe implantation.
\end{abstract}

\section{Introduction}

Radiotherapy is a major treatment for head and neck cancers. However, irradiation can have a negative impact on oral cavity homeostasis. ${ }^{1,2}$ Side effects or complications implicate teeth, supporting tissue mucous membranes, salivary glands, and muscles. ${ }^{1-4}$ Radio-induced hypovascularity provokes hypoxia in radio-induced hypocellular tissues. ${ }^{5}$ The ability of these irradiated tissues to respond suitably to physiologic and nonphysiologic stressors is reduced and depends on the irradiated volume and the delivered dose. Indeed, aggressiveness of postirradiation dental care must be prevented because of the weakness of these tissues. ${ }^{1,2,6-16}$ In this context, knowledge of dose distribution is key to safely 
managing dental care. ${ }^{17}$ Thanks to dose-volume histograms in healthy tissues, risks of complications can be predicted and dental care can be adapted. ${ }^{18,19}$ However, if the locations of required implants could be determined before irradiation, radiation oncologists could validate the dosimetry after considering the shielding of the bone where the implants should be fixed.

In this study, based on data from 43 patients who were irradiated for a head and neck lesion, the dentist prospectively proposed dental rehabilitation with different types of prosthodontic treatment, eventually including implants. During dosimetry, the radiation oncologist was blinded to the dental rehabilitation plan. Then, the authors analyzed whether the dentist's proposals before dosimetry calculation remained suitable after calculation of the dose distribution in the mandible and maxilla.

\section{Materials and Methods}

The study was approved by the consultative board of research of the hospital. About $70 \%$ of the tumors were localized to the mouth cavity, oropharynx, hypopharynx, and larynx. All patients were irradiated in the lymph nodes areas with prophylactic or curative intents. After computed tomography scans with or without contrast injection, the target volume and critical organs including the mandible and maxilla were delimited according to a previously published method ${ }^{20}$ using Focal software (CMS, XiO, Elekta AB, Sweden). Briefly, the maxilla and mandible were virtually divided into three sextants each. The use of sextants simplifies the shielding of parts of the jaw. Irradiation was delivered with intensity modulated radiation therapy (IMRT). The median prescribed dose was 66 Gy (mean: 61.2 Gy; range: 56-70) with a daily dose of $2 \mathrm{~Gy}$ at five fractions per week. The mean and maximal doses in the sextants were calculated using Artiview software (Artiview Dose, Aquilab SAS, Loos, France).

\section{Description of Edentulous Patients}

The types of edentulous patients are described in - Fig. $\mathbf{1}$. Considering all of the sextants, significantly more lateral sextants than anterior sextants had missing teeth $(p<0.001)$. Complete dentates (CDSs), partially edentulous (PESs), and completely edentulous sextant (CES) distributions significantly differed whether they were lateral or anterior $(p<0.001)$. There was no difference between the types of edentulous sextants according to the tumor locations.

\section{Prosthesis Rehabilitation Completely Edentulous Arches}

In the seven completely edentulous patients (CEPs), two patients had removable complete denture (RCDs) for both the maxilla and the mandible; two patients had not undergone prosthetic rehabilitation. For three patients, extractions of their remaining teeth were performed, and additions of fake teeth to their previous RCDs or an immediate RCD were done.

For the nonrehabilitated CEPs, RCDs were always suitable. In the mandible, to improve stability and retention of these RCDs, two implants on the anterior sextant are usually proposed (stabilized RCDs [SRCDs]). In the maxilla, a minimum of four implants on three sextants is necessary

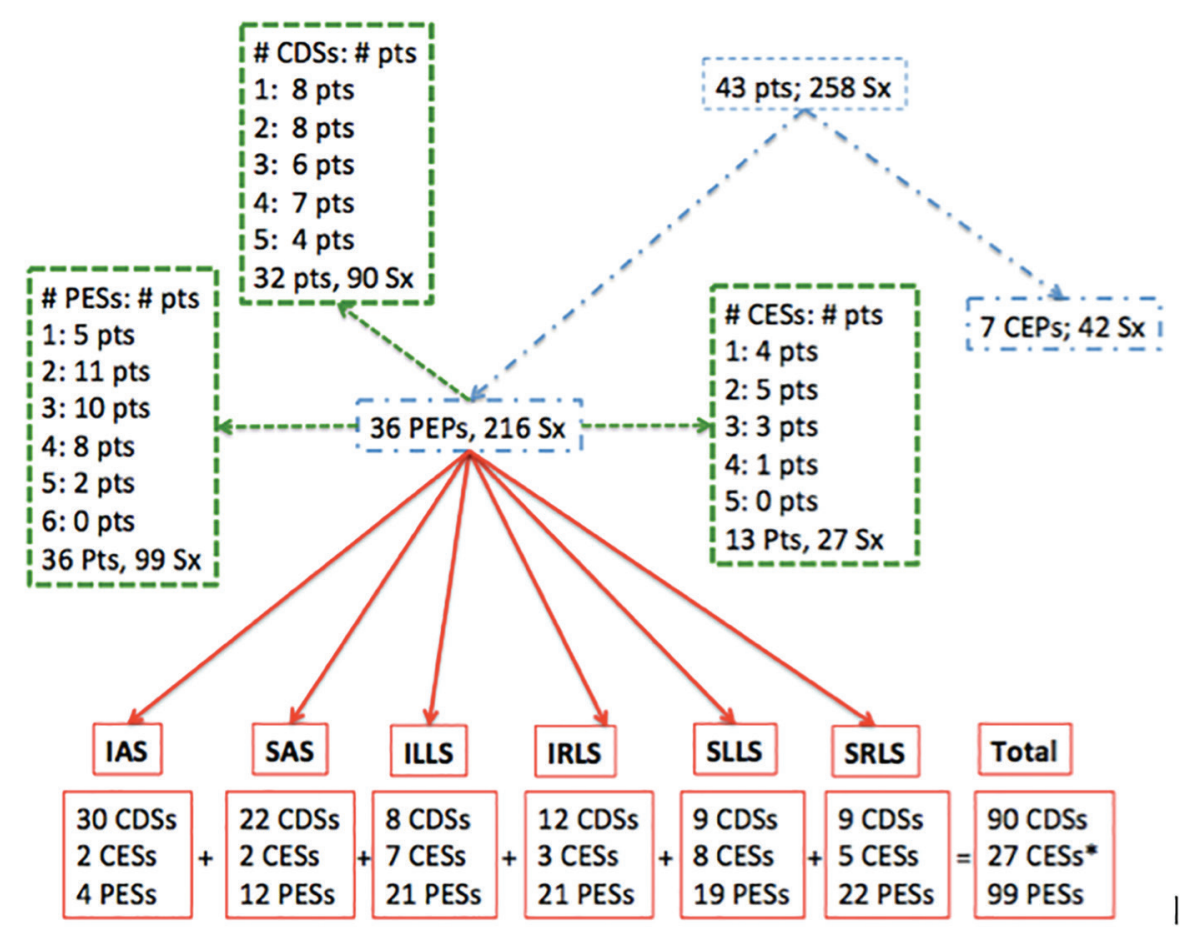

Fig. 1 Distribution of the patients or sextants according to their dental status. CD: Complete dentate (patient [P] or sextant [S]); PE: Partially edentulous (patient [P] or sextant [S]); CE: Complete edentulous (patient [P] or sextant [S]), pts: Patients. *Among the 27 sextants, 5 sextants in hemimandibulectomies for 4 patients. IAS: Inferoanterior sextant; ILLS: Inferior and left lateral sextant; IRLS: Inferior and right lateral sextant; SAS: Supero-anterior sextant; SLLS: Superior and left lateral sextant; SRLS: Superior and right lateral sextant; Sx: Sextant. 
to realize an SRCD. When the quality of the bone anatomy and support tissues allowed it, a fixed prosthesis over implants (FPI) could be proposed. To achieve efficient fixation of this prosthesis, at least two implants are required in each sextant of the maxilla or the mandible. Theoretically, before irradiation, all CEPs could have an FPI or an SRCD.

\section{Partially Edentulous Patients and Arches}

Among the 216 segments of the 36 PESs patients, 3 arches were completely edentulous and the prosthesis proposals and possibilities after dosimetry have been considered equivalent to the cases of CEPs.

Among the other 207 segments, 113 did not require implant rehabilitation for the following reasons: CDSs, crowns (that could be replaced by bridges without implants), or sextants already fixed with adapted implanted prostheses.

The remaining 94 segments required rehabilitation. Among them, 34 segments were rehabilitated, but the results were not adapted and new proposals were made. For the last 60 segments, any rehabilitation was performed at the time of the dentist consultation. The main proposal was an FPI. If the patient could not be fixed with an FPI, either a removable partial denture or a bridge could be proposed, but only the FPI was implanted for rehabilitation.

\section{Dosimetry Analysis}

For CDPs and PEPs, for the risk of implant complications, the authors calculated the dose received by the bone of each sextant where there were remaining teeth for dental care and where an implant could be fixed.

\section{Risk of Failure of Implant}

According to the literature, three classes of implant failure risk have been specified, strictly $<40 \mathrm{~Gy}$, the risk is low at almost $0 \%$; from 40 to $50 \mathrm{~Gy}$, the risk is intermediate; and at more than $50 \mathrm{~Gy}$, the risk is high and surgery must not be undertaken. ${ }^{21,22}$

Before dosimetry, the dentist proposed prosthetic rehabilitations for each patient, but the radiation oncologist did not know the proposals before planning treatment validation. Once the treatment planning was validated, the authors evaluated the feasibility of implant-carried prostheses depending on the risk.

\section{Statistics}

Comparisons by Levene's test or by a single-factor ANOVA test were used. All the calculations were performed using SPSS software, version 22.0 (IBM corporation, Armonk, New York, United States).

\section{Results}

\section{Prosthetic Proposals and Received Dose}

This risk is related to the portion of the sextants where teeth are missing. There were 42 CESs for the 7 CEPs and 27 CESs, and 99 PESs for the 36 PEPS. Anterior sextants were significantly less edentulous than lateral sextants: 34 sextants (39\%)and 134 sextants (78\%), respectively ( Fig. 1) $(p<0.0001)$.
According to mean dose, the sextant rates in the three groups for dose at low, intermediate, and high risks were $60.7 \%, 20.6 \%$, and $18.4 \%$, respectively. For the maximal dose, these distribution rates were $37.4 \%, 12.3 \%$, and $50.3 \%$, respectively. The mean dose of PESs was significantly more frequently $>40$ Gy than that of CESs $(p<0.01)$, but the distribution of the maximal dose was not significantly different. The highest dose was significantly more frequently delivered to the lateral sextants than the anterior sextant $(p<0.001)$. However, there was no significant difference in dose distributions (for mean and maximal doses) according to the bone location (mandibular or maxillary).

\section{Completely Edentulous Patients}

Before calculation all 14 of the arches of the 7 CEPs could be optimally indifferently implanted with FPI or SRCD.

According to the mean doses after dose calculation, 11 arches (78.6\%) could receive an optimal prosthesis; for 8 arches, FPI could be implanted at low risk; for one arch, SRCD could be implanted at low risk or FPI at intermediate risk; and for the two remaining arches, SRCD could be implanted at low risk but FPI at high risk. The three remaining arches could not receive optimal prostheses: for one arch, FPI or SRCD could be implanted with intermediate risk for both types of implantation, and two arches could receive neither FPI nor SRCD because of high risk for both implantations. In total, four patients could receive optimal prostheses in both arches, and the three remaining patients could receive an optimal prosthesis in only one arch.

According to the maximal dose, 7 arches (50\%) could receive an optimal prosthesis. For 3 arches, FPI could be implanted at low risk; for one arch, SRCD could be implanted at low risk or FPI at intermediate risk; and for the three remaining arches, SRCD could be implanted at low risk but FPI at high risk. Seven remaining arches could not receive optimal prostheses: for one arch, FPI or SRCD could be implanted with intermediate risk for both types of implantation; for one arch, SRCD could be implanted at intermediate risk but FPI at high risk; and five arches could receive neither FPI nor SRCD because of high risk for both implantations. In total, two patients could receive optimal prostheses in both arches, three could receive an optimal prosthesis in only one arch, and two patients could not be implanted optimally in either of their arches.

For the three PEPs with one $\mathrm{CE}$ arch, according to the mean doses, one arch could be improved with an SRCD at low risk and an FPI at intermediate risk, and one arch could be improved by either an SRCD or an FPI but the two with intermediate risk and the last could not receive any improvement because of the high risk to implant an SRCD or an FPI.

According to the maximal doses, one arch could be improved with an SRCD at low risk but not with an FPI because of the high dose, and two arches could not receive any improvement because of the high risk of implanting an SRCD or an FPI. 


\section{Partially Edentulous}

Among the 94 PESs imperfectly fixed or nonimplanted before radiotherapy, all could achieve optimally implanted rehabilitation.

Among the PESs, 18 segments in the maxilla and 16 in the mandible were fixed with a nonadapted prosthesis in place before radiotherapy. According to the mean dose, 22 prostheses could be improved at low risk, 10 could be improved at intermediate risk, and two could not be improved at all. According to maximal dose, the figures were 10,10 , and 14 , respectively, for low-, intermediate-, and high-risk rehabilitation.

Among the PESs, 25 segments in the maxilla and 35 in the mandible should have been implanted, but they were not before radiotherapy. According to the mean dose, 19 prostheses could be improved at low risk, 20 could be improved at intermediate risk, and 21 could not be improved at all. According to maximal dose, the figures were 14, 25, and 21 , respectively, for low-, intermediate-, and high-risk rehabilitation.

In total, among the 94 PESs that could be rehabilitated, according to the mean dose, 41 (43.6\%) could be implanted a low risk, 30 (31.9\%) could be rehabilitated at intermediate risk, and $23(24.5 \%)$ could not be implanted because of high risk. However, if the maximal dose is considered, the number of sextants according to the rehabilitation risk was 24 (25.5\%), 35 (37.25\%), and 35 (37.25\%), respectively.

\section{Discussion}

Surgery and irradiation are the main treatments for head and neck carcinoma. After completion of treatment and control of disease, rehabilitation and restoration of function are challenging tasks. The presence of dentition is one of the main components for fabrication of a retentive and stable dental prosthesis, which restores oral functions such as mastication, swallowing, aesthetics, and speech. These problems greatly decrease the quality of life of patients treated for head and neck cancers compared to the normal population. ${ }^{23}$ Dental rehabilitation is an important factor in improvement of quality of life and eating..$^{24,25}$

Dental implants contribute to the feasibility of fabrication of implant-retained dental prostheses in patients with head and neck cancer, facilitating restoration of oral functions. Over the last past three decades, the use of implants for rehabilitation has dramatically increased owing to advancements in materials and surgical procedures. ${ }^{26}$ The impact of dentures on quality of life are ambiguous because, although a positive quality of life score is often associated with fixed partial denture or dentures with optimal retention, ${ }^{27,28}$ a recent study showed that type of rehabilitation seems have no impact on the quality of life of patients with head and neck tumors. ${ }^{23}$ As a consequence, after rehabilitation, dentists cannot assume the same expectation about quality of life in patients with head and neck cancer compared to the normal population. ${ }^{27-29}$

In patients with oral cancer, the effect of radiation therapy on the vascular and cellular components of the tissues and altered anatomy due to reconstructions of bone are important determinants of osseointegration and overall success of implants. Radiotherapy can induce changes in bone by reducing the vascularity and regenerative ability of the tissue. Hypovascularization is the consequence of increased bone mineral density leading to bone sclerosis. ${ }^{30}$ Some events generate imbalances between bone formation and resorption. Indeed, bone formation is affected because osteoblasts and osteocytes lose their bone-forming ability and osteoclasts, which are responsible for bone resorption, are attracted to irradiated sites. ${ }^{31,32}$ With time changes in bones exhibit marked acellularity, avascularity, fibrosis, and fatty degeneration..$^{33}$ Dental implant failure has rarely been observed at a cumulative dose $<45 \mathrm{~Gy},{ }^{21,34}$ and some authors have argued that the failure rate in patients with a cumulative dose $<50 \mathrm{~Gy}$ in the maxilla or mandible is comparable to that in patients who have not undergone radiation therapy ${ }^{35-37}$ However, the survival of implants is clearly shortened if cumulative doses are greater than $50-55 \mathrm{~Gy},{ }^{34,38,39}$ and failure is very common at $65 \mathrm{~Gy} .{ }^{34}$ Thus, reports have indicated that value thresholds at 50-60 Gy are the borderline for rehabilitation with dental implants without the requirement of additional treatment. ${ }^{37,38,40,-44}$ These values are very close to those used to sterilize tumors since a dose of $50 \mathrm{~Gy}$ is classically delivered in an adjuvant procedure and $70 \mathrm{~Gy}$ for exclusive irradiation. According to results in dogs, some teams did not advise implants when doses greater than $50 \mathrm{~Gy}$ have been delivered..$^{23,45} \mathrm{~A}$ recent meta-analysis demonstrated the definitive role of irradiated tissues in the failure of dental implants, with a risk of $15.5 \%$ of failure in irradiated tissues compared to $5.2 \%$ in nonirradiated bone $(p<0.001){ }^{46}$ In this study, we split the doses delivered to bone, into three groups: $<40 \mathrm{~Gy}$, 40-50 Gy, and > 50 Gy, representing low, intermediate, and high risks of implant failure, respectively.

Some studies have shown that mandible holds implants more efficiently than the maxilla, ${ }^{47}$ likely because of a larger cortical bone improving stability and better bone quality and vascularization ability, both participating in bone resistance. ${ }^{26}$ Furthermore, the fact that mandibular bone has more trabecular structure results in the participation of better vascularization in this bone. ${ }^{48}$ Notably, in this study, we showed that the mean delivered irradiation dose did not differ according to the two arches but the doses were significantly lower in the anterior sextants of the two arches, emphasizing that the role of the dose depends on the bone receiving irradiation. This point was reported in a recent review ${ }^{49}$ using the same delineation guidelines, ${ }^{20}$ O'Cathail et al showed comparable results..$^{50}$ Coincidently, these sites are also areas where more teeth are missing. In this series, the edentulous distribution was significantly different according to the sextants, with $64.6 \%$ of CDSs in anterior sextants, compared to $30.2 \%$ in lateral sextants. In our study, this difference in dose is the consequently edentulous sextant of the bone was not due to the desire to protect bone but the goal of limiting mucositis. For this reason, radiation oncologists avoid using beams that pass through the oral cavity. Visch et al observed a difference in 
implant survival between anterior and posterior implantation, but the difference was not significant. ${ }^{38}$

To decrease the risk of implant failure, hyperbaric oxygen (HBO) therapy is currently used..$^{51}$ The authors have shown a dramatically decrease in the risk, from approximately $54 \%$ to $8 \%$, using the HBO therapy. ${ }^{52}$ However, the results remain disputable since studies have not shown any improvement. ${ }^{44,53}$ Furthermore, the protocol is relatively restrictive, requiring 20-30 sessions of $90 \mathrm{~min}$ at a compression of 2.4 atmospheres of absolute pressure with $100 \%$ oxygen. ${ }^{54}$ Furthermore, limited accessibility of the device, the cost of the procedure, and the risks of ear barotraumas or myopia are a factor in HBO not being universally accepted in dentistry. ${ }^{44,49,53}$

Being less aggressive, the knowledge of the dose in the potential site of the implant before implantation could be very useful and could provide helpful information to the dentists. Delineation of the mandible and maxilla into sextants, affording the opportunity to specify doses in specific sites can participate in preventing risk. ${ }^{20}$ This ideal was reported by Tanaka et al in their review of clinical considerations for dental implantations. ${ }^{49}$

To the best of our knowledge, this study was the first prospective study that attempted to correlate the initial proposals for dental rehabilitation with the effective possible rehabilitation after delivered doses to the mandible and the maxilla.

At the time of three-dimensional (3D) irradiation, the main difficulty for the radiation oncologist is to know precisely the dose constraints to the maxillary and mandibular to avoid as much as possible the risk of complication. Indeed, it was very difficult to spare some parts of jaws, mainly the posterior part of the mandible, without protecting a part of the tumor. ${ }^{55}$ Today, with the IMRT, radiation oncologists can impose some dose constraints on the bone tissues, and algorithms can calculate dose distributions both to deliver adequate doses to the tumor and to follow the constraints on the critical organs without negative compromises. In the previously published studies, it was difficult to determine whether the reported doses were mean or maximum doses and whether these doses were those delivered in the place of implantation or the total mandible or maxilla. By delineation, it is possible to determine retrospectively the dose received by the bone where the dentist proposes to fix an implant. Furthermore, as demonstrated by this study, the dentist should report the site where he or she wants to implant because, in the absence of these data, some potential sites available for implantation can become unsuitable after irradiation because of the delivered doses.

For the authors, the dose and time of implantation are not prognostic factors for implant survival: only the site of placement is a relevant factor. ${ }^{47}$ However, as shown in this study, there is a correlation between sextant and the received dose. Although in our series, there was no difference in dose distribution in the mandibular arch and maxillary arch to support the role of dose in failure according to both the localizations in the jaw, there was a difference between anterior and lateral sextants, making an argument for considering dose to be a relevant factor in decreasing failure rates. Bone surgery may be performed only in healthy and low-irradiated bone. Two solutions can be proposed: providing the dentist with a precise dosimetry distribution of the bone $\mathrm{e}^{20}$ and preventing risk by shielding the bone structure, which would require surgical management after irradiation. ${ }^{56,57}$ To reach the latter solution, our study showed that not sharing the dentist's data or the dentist not being involved in the proposals leads to a lack of opportunity to attain optimal rehabilitation. Indeed, according to the mean doses delivered among the sextants as sites of a possible rehabilitation, $47.7 \%$ could be optimally implanted, i.e., at a low-risk dose. According to the maximal dose, this rate dropped to $27.9 \%$. Thus, a discussion before planning treatment validation could reduce the risk of implant failure, mainly if the radiation oncologist could avoid intermediate and high-risk doses, especially in the missing tooth areas. Today, with the development of IMRT, the radiation oncologist can adapt doses for the maxilla and the mandible sextants, provided that there is awareness of the implant proposal before dose calculation. One small study suggested that it is dosimetrically possible to spare the anterior mandible using IMRT. However, the authors measured only five points between the right and left mental foramen, ${ }^{58}$ although we calculated doses in 3D in 6 sextants.

Dental implants in irradiated bone in patients treated for head and neck cancers are a controversial subject. According to some authors, irradiation is a contraindication in implantation because of the radionecrosis risk. In a recent review of the practice in Spain, the authors evoked the implants only to measure the high risk of fixing them. Prevention to succeed with these implants was not discussed. ${ }^{59}$ However, analyses in the literature have suggested that complication incidence is not significantly greater than in healthy bone., ${ }^{71,18,19,21,60-63}$ These controversies again highlight the necessity for dialogue between the radiation oncologists and the dentist upstream of the radiotherapy. Furthermore, Chambrone et al indicated that dental implants are an effective treatment that often permanently restores the occlusion and mastication in patients who have undergone radiation therapy, even if the associated implant failure rate is higher in an irradiated bone compared to a healthy bone. ${ }^{60}$ The authors concluded that the most important data were the anatomical sites of implantation, with the best results in the mandible (93.3\%) rather than the maxilla (78.9\%). ${ }^{60}$ More than the complete arches, our study suggested that the ability to restore is related to the sextant. Loss of possibility to implant is clearly demonstrated by doses delivered in not shielded sextants. According to the mean dose, among 6 impossible FPIs in the CE arches, five were the consequences of high doses in lateral sextants compared to one for the anterior sextants; according to the maximal dose among 11 arches unsuitable for an FPI, only three cases were the consequences of high-risk doses in anterior sextants. For the PEPs, according to the mean dose, there were three that were impossible to rehabilitate because of the high-risk dose being localized in lateral sextants, and according to the maximal dose, among the 19 impossible rehabilitations, only two were secondary to the 
dose in an anterior sextant. In our study, we showed that lack of implantation site indication lead to losing the opportunity to fix an implant accurately because of the delivered dose being too high. In another approach, using the similar delineation guideline, ${ }^{20}$ O'Cathail et al replanned patients who received more than $37.5 \mathrm{~Gy}$ in the anterior sextant of the mandible. They succeeded in decreasing the dose to less than this threshold in $88 \%$ of the patients without breaching accepted organs at risk constraints and without degradation of the dose distribution into the tumor..$^{50}$ Integration of consultation into restorative dentistry as core members of the multidisciplinary team treating head and neck cancer patients is absolutely required..$^{51}$

Nevertheless, knowing the implantation sites and to be able to limit the dose in the relevant sites might not be sufficient to avoid bone complications. Indeed, to decrease the dose in some areas requires increasing it in other sites, mainly where there are healthy teeth; thus, this potentially increases the risk of complications. This must be prospectively analyzed for future routine dental care.

\section{Conclusion}

Our study showed that the ability for rehabilitation is related to the doses delivered in the jaw. Our study also suggests that a close discussion between the radiation oncologist and the dentist upstream of the dosimetry is required to attempt to improve the possibility of postirradiation dental rehabilitation.

\section{Financial Support and Sponsorship}

None.

\section{Conflicts of Interest}

There are no conflicts of interest.

\section{References}

1 Andrews N, Griffiths C. Dental complications of head and neck radiotherapy: Part 2. Aust Dent J 2001;46(3):174-182

2 Andrews N, Griffiths C. Dental complications of head and neck radiotherapy: Part 1. Aust Dent J 2001;46(2):88-94

3 Eisbruch A, Ten Haken RK, Kim HM, Marsh LH, Ship JA. Dose, volume, and function relationships in parotid salivary glands following conformal and intensity-modulated irradiation of head and neck cancer. Int J Radiat Oncol Biol Phys 1999;45(3):577-587

4 Kałużny J, Wierzbicka M, Nogala H, Milecki P, Kopeć T. Radiotherapy induced xerostomia: mechanisms, diagnostics, prevention and treatment-evidence based up to 2013. Otolaryngol Pol 2014;68(1):1-14

5 Marx RE. Osteoradionecrosis: a new concept of its pathophysiology. J Oral Maxillofac Surg 1983;41(5):283-288

6 Beech N, Robinson S, Porceddu S, Batstone M. Dental management of patients irradiated for head and neck cancer. Aust Dent J 2014;59(1):20-28

7 Buddula A, Assad DA, Salinas TJ, Garces YI. Survival of dental implants in native and grafted bone in irradiated head and neck cancer patients: a retrospective analysis. Indian J Dent Res 2011;22(5):644-648

8 Buddula A, Assad DA, Salinas TJ, Garces YI, Volz JE, Weaver AL. Survival of dental implants in irradiated head and neck cancer patients: a retrospective analysis. Clin Implant Dent Relat Res 2012;14(5):716-722
9 Claudy MP, Miguens SA Jr, Celeste RK, Camara Parente R, Hernandez PA, da Silva AN. Time interval after radiotherapy and dental implant failure: systematic review of observational studies and meta-analysis. Clin Implant Dent Relat Res 2015;17(2):402-411

10 Devi S, Singh N. Dental care during and after radiotherapy in head and neck cancer. Natl J Maxillofac Surg 2014;5(2):117-125

11 Dholam KP, Gurav SV. Dental implants in irradiated jaws: a literature review. J Cancer Res Ther 2012;8(Suppl 1):S85-S93

12 Jaussaud S, Guihard S, Niederst C, et al. Les contraintes du chirurgien dentiste sont compatibles avec les résultats d'une irradiation optimale avec modulation d'intensité dans les cancers de l'oropharynx de stade N0. [Constraints of the dentist are consistent with the results of an optimal irradiation with modulated intensity in N0 oropharyngeal cancer]. Cancer Radiother 2013;17(4):265-271

13 Regezi JA, Courtney RM, Kerr DA. Dental management of patients irradiated for oral cancer.Cancer 1976;38(2):994-1000

14 Rouers M, Truntzer P, Dubourg S, Guihard S, Antoni D, Noël G. État dentaire des patients atteints d'un cancer des voies aérodigestives supérieures. [Dental state in patients with head and neck cancers]. Cancer Radiother 2015;19(3):205-210, quiz 230, 234

15 Thariat J, De Mones E, Darcourt V, et al. Dent et irradiation: denture et conséquences sur la denture de la radiothérapie des cancers de la tête et du cou. [Teeth and irradiation in head and neck cancer]. Cancer Radiother 2010;14(2):128-136

16 Thariat J, de Mones E, Darcourt V, et al. Dent et irradiation: prévention et traitement des complications dentaires de la radiothérapie y compris l'ostéoradionécrose. [Teeth and irradiation: dental care and treatment of osteoradionecrosis after irradiation in head and neck cancer].Cancer Radiother 2010;14(2):137-144

17 Mérigot A, Chatel A. Irradiation cervico-faciale: répercussion sur le milieu buccal. Rev Odontostomatol (Paris) 2005; 34:155-169

18 Javed F, Al-Hezaimi K, Al-Rasheed A, Almas K, Romanos GE. Implant survival rate after oral cancer therapy: a review. Oral Oncol 2010;46(12):854-859

19 Mancha de la Plata M, Gías LN, Díez PM, et al. Osseointegrated implant rehabilitation of irradiated oral cancer patients. J Oral Maxillofac Surg 2012;70(5):1052-1063

20 Rouers M, Antoni D, Thompson A, et al. Maxillary and mandible contouring in patients with a head and neck area irradiation. Pract Radiat Oncol 2016;6(3):e61-e72

21 Colella G, Cannavale R, Pentenero M, Gandolfo S. Oral implants in radiated patients: a systematic review. Int J Oral Maxillofac Implants 2007;22(4):616-622

22 Hindorf C, Glatting G, Chiesa C, Lindén O, Flux G; EANM Dosimetry Committee. EANM Dosimetry Committee guidelines for bone marrow and whole-body dosimetry. Eur J Nucl Med Mol Imaging 2010;37(6):1238-1250

23 Schweyen R, Kuhnt T, Wienke A, Eckert A, Hey J. The impact of oral rehabilitation on oral health-related quality of life in patients receiving radiotherapy for the treatment of head and neck cancer. Clin Oral Investig 2017;21(4):1123-1130

24 Jacobsen HC, Wahnschaff F, Trenkle T, Sieg P, Hakim SG. Oral rehabilitation with dental implants and quality of life following mandibular reconstruction with free fibular flap. Clin Oral Investig 2016;20(1):187-192

25 Shigli K, Hebbal M. Does prosthodontic rehabilitation change the eating patterns among completely edentulous patients? Gerodontology 2012;29(1):48-53

26 Granström G. Osseointegration in irradiated cancer patients: an analysis with respect to implant failures. J Oral Maxillofac Surg 2005;63(5):579-585 
27 Hassel AJ, Koke U, Schmitter M, Rammelsberg P. Factors associated with oral health-related quality of life in institutionalized elderly. Acta Odontol Scand 2006;64(1):9-15

28 John MT, Slade GD, Szentpétery A, Setz JM. Oral health-related quality of life in patients treated with fixed, removable, and complete dentures 1 month and 6 to 12 months after treatment. Int J Prosthodont 2004;17(5):503-511

29 Dawood A, Tanner S. 'Sometimes the simplest interventions can make a big difference'. Br Dent J 2013;214(5):261-263

30 Verdonck HW, Meijer GJ, Nieman FH, Stoll C, Riediger D, de Baat C. Quantitative computed tomography bone mineral density measurements in irradiated and non-irradiated minipig alveolar bone: an experimental study. Clin Oral Implants Res 2008;19(5):465-468

31 Bolind P, Johansson CB, Johansson P, Granström G, Albrektsson T. Retrieved implants from irradiated sites in humans: a histologic/histomorphometric investigation of oral and craniofacial implants. Clin Implant Dent Relat Res 2006;8(3):142-150

32 Brogniez V, Lejuste P, Pecheur A, Reychler H. Dental prosthetic reconstruction of osseointegrated implants placed in irradiated bone. Int J Oral Maxillofac Implants 1998;13(4):506-512

33 Marx RE, Johnson RP. Studies in the radiobiology of osteoradionecrosis and their clinical significance. Oral Surg Oral Med Oral Pathol 1987;64(4):379-390

34 Granström G. Placement of dental implants in irradiated bone: the case for using hyperbaric oxygen. J Oral Maxillofac Surg 2006;64(5):812-818

35 Garrett N, Roumanas ED, Blackwell KE, et al. Efficacy of conventional and implant-supported mandibular resection prostheses: study overview and treatment outcomes. J Prosthet Dent 2006;96(1):13-24

36 Larsen PE. Placement of dental implants in the irradiated mandible: a protocol involving adjunctive hyperbaric oxygen. J Oral Maxillofac Surg 1997;55(9):967-971

37 Sammartino G, Marenzi G, Cioffi I, Teté S, Mortellaro C. Implant therapy in irradiated patients. J Craniofac Surg 2011;22(2):443-445

38 Visch LL, van Waas MA, Schmitz PI, Levendag PC. A clinical evaluation of implants in irradiated oral cancer patients. J Dent Res 2002;81(12):856-859

39 Yerit KC, Posch M, Seemann M, et al. Implant survival in mandibles of irradiated oral cancer patients. Clin Oral Implants Res 2006;17(3):337-344

40 Andersson G, Andreasson L, Bjelkengren G. Oral implant rehabilitation in irradiated patients without adjunctive hyperbaric oxygen. Int $\mathrm{J}$ Oral Maxillofac Implants 1998;13(5):647-654

41 August M, Bast B, Jackson M, Perrott D. Use of the fixed mandibular implant in oral cancer patients: a retrospective study. J Oral Maxillofac Surg 1998;56(3):297-301

42 Dholam KP, Pusalkar HA, Yadav PS, Quazi GA, Somani PP. Implant-retained dental rehabilitation in head and neck cancer patients: an assessment of success and failure. Implant Dent 2013;22(6):604-609

43 Jisander S, Grenthe B, Alberius P. Dental implant survival in the irradiated jaw: a preliminary report. Int J Oral Maxillofac Implants 1997;12(5):643-648

44 Shaw RJ, Sutton AF, Cawood JI, et al. Oral rehabilitation after treatment for head and neck malignancy. Head Neck 2005;27(6):459-470

45 Asikainen P, Klemetti E, Kotilainen R, et al. Osseointegration of dental implants in bone irradiated with 40,50 or $60 \mathrm{~Gy}$ doses. An experimental study with beagle dogs. Clin Oral Implants Res 1998;9(1):20-25
46 Smith Nobrega A, Santiago JF Jr, de Faria Almeida DA, Dos Santos DM, Pellizzer EP, Goiato MC. Irradiated patients and survival rate of dental implants: a systematic review and meta-analysis. J Prosthet Dent 2016;116(6):858-866

47 Zen Filho EV, Tolentino EdeS, Santos PS. Viability of dental implants in head and neck irradiated patients: a systematic review. Head Neck 2016;38(Suppl 1):E2229-E2240

48 Verdonck HW, Meijer GJ, Laurin T, et al. Implant stability during osseointegration in irradiated and non-irradiated minipig alveolar bone: an experimental study. Clin Oral Implants Res 2008;19(2):201-206

49 Tanaka TI, Chan HL, Tindle DI, Maceachern M, Oh TJ. Updated clinical considerations for dental implant therapy in irradiated head and neck cancer patients. J Prosthodont 2013;22(6):432-438

50 O'Cathail SM, Karir N, Shah K. Optimising volumetric arc radiotherapy for dental rehabilitation in oropharynx cancer-a retrospective dosimetry review and feasibility planning study. Oral Oncol 2018;76:16-21

51 Butterworth C, McCaul L, Barclay C. Restorative dentistry and oral rehabilitation: United Kingdom National Multidisciplinary Guidelines. J Laryngol Otol 2016;130(S2) :S41-S44

52 Granström G, Tjellström A, Brånemark PI. Osseointegrated implants in irradiated bone: a case-controlled study using adjunctive hyperbaric oxygen therapy. J Oral Maxillofac Surg 1999;57(5):493-499

53 Schoen PJ, Raghoebar GM, Bouma J, et al. Rehabilitation of oral function in head and neck cancer patients after radiotherapy with implant-retained dentures: effects of hyperbaric oxygen therapy. Oral Oncol 2007;43(4):379-388

54 Marx RE, Johnson RP, Kline SN. Prevention of osteoradionecrosis: a randomized prospective clinical trial of hyperbaric oxygen versus penicillin. J Am Dent Assoc 1985;111(1):49-54

55 Kufta K, Forman M, Swisher-McClure S, Sollecito TP, Panchal N. Pre-radiation dental considerations and management for head and neck cancer patients. Oral Oncol 2018;76:42-51

56 Jawad H, Hodson NA, Nixon PJ. A review of dental treatment of head and neck cancer patients, before, during and after radiotherapy: part 2. Br Dent J 2015;218(2):69-74

57 Jawad H, Hodson NA, Nixon PJ. A review of dental treatment of head and neck cancer patients, before, during and after radiotherapy: part 1. Br Dent J 2015;218(2):65-68

58 Verdonck HW, de Jong JM, Granzier ME, Nieman FH, de Baat C, Stoelinga PJ. Intensity-modulated radiation therapy for oropharyngeal cancer: radiation dosage constraint at the anterior mandible. Oral Oncol 2009;45(6):511-514

59 Lanzós I, Herrera D, Lanzós E, Sanz M. A critical assessment of oral care protocols for patients under radiation therapy in the regional University Hospital Network of Madrid (Spain). J Clin Exp Dent 2015;7(5):e613-e621

60 Chambrone L, Mandia J Jr, Shibli JA, Romito GA, Abrahao M. Dental implants installed in irradiated jaws: a systematic review. J Dent Res 2013;92(12, Suppl):119S-130S

61 Ihde S, Kopp S, Gundlach K, Konstantinović VS. Effects of radiation therapy on craniofacial and dental implants: a review of the literature. Oral Surg Oral Med Oral Pathol Oral Radiol Endod 2009;107(1):56-65

62 Kwakman JM, Freihofer HP, van Waas MA. Osseointegrated oral implants in head and neck cancer patients. Laryngoscope 1997;107(4):519-522

63 Schoen PJ, Reintsema H, Raghoebar GM, Vissink A, Roodenburg JL. The use of implant retained mandibular prostheses in the oral rehabilitation of head and neck cancer patients. A review and rationale for treatment planning. Oral Oncol 2004;40(9):862-871 